\title{
Retrospective Comparison of Long-Term Clinical Outcomes Between Percutaneous Coronary Intervention and Medical Therapy in Stable Coronary Artery Disease With Gray Zone Fractional Flow Reserve - COMFORTABLE Retrospective Study -
}

\author{
Takashi Kubo, MD, PhD; Masahiro Takahata, MD; Kosei Terada, MD; \\ Kazuya Mori, MD; Yu Arita, MD; Yasushi Ino, MD, PhD; \\ Yoshiki Matsuo, MD, PhD; Hironori Kitabata, MD, PhD; Yasutsugu Shiono, MD, PhD; \\ Kunihiro Shimamura, MD, PhD; Takeyoshi Kameyama, MD, PhD; Hiroki Emori, MD, PhD; \\ Yosuke Katayama, MD; Takashi Tanimoto, MD, PhD; Takashi Akasaka, MD, PhD
}

Background: A fractional flow reserve (FFR) between 0.75 and 0.80 constitutes a "gray zone" for clinical decision-making in coronary artery disease. We compared long-term outcomes of percutaneous coronary intervention (PCl) using drug-eluting stents vs. medical therapy for coronary stenosis with gray zone FFR.

Methods and Results: We retrospectively investigated the clinical outcomes of 263 patients with gray zone FFR: 78 patients in the $\mathrm{PCl}$ group and 185 patients in the medical therapy group. During a median follow-up of 3.7 years, the frequency of target vessel failure (TVF, defined as a composite of cardiac death, myocardial infarction [MI], or ischemia-driven target vessel revascularization [TVR]) was significantly lower in the PCl group compared with the medical therapy group (6\% vs. 19\%, hazard ratio [HR]:0.33, 95\% confidence interval [Cl]: $0.13-0.84, \mathrm{P}=0.008)$. The frequency of a composite of cardiac death or MI was not different between the 2 groups ( $1 \%$ vs. $2 \%$, HR: $0.61,95 \% \mathrm{Cl}: 0.07-5.49, \mathrm{P}=0.645)$. The frequency of ischemia-driven TVR was significantly lower in the $\mathrm{PCl}$ group compared with the medical therapy group (5\% vs. $18 \%, \mathrm{HR}: 0.28,95 \% \mathrm{Cl}: 0.10-0.79, \mathrm{P}=0.005)$.

Conclusions: In patients with gray zone FFR, compared with medical therapy, PCl decreased the frequency of TVF, which was mainly driven by a reduction in the frequency of angina or myocardial ischemia without any difference in the frequency of cardiac death or MI.

Key Words: Coronary physiology; Drug-eluting stents; Fractional flow reserve; Percutaneous coronary intervention

$\mathbf{F}$ ractional flow reserve (FFR) is an invasive index used to determine the functional significance of coronary stenoses. An FFR $<0.75$ is associated with myocardial ischemia. ${ }^{1}$ The DEFER (Deferral vs. performance of percutaneous coronary intervention of functionally non-significant coronary stenosis) study demonstrated the safety of deferral of percutaneous coronary intervention (PCI) in coronary stenoses with FFR $\geq 0.75 . .^{2}$ In contrast, an FFR $\leq 0.80$ is an indication for revascularization. The FAME 2 (Fractional flow reserve vs. angiography for multivessel evaluation 2) study, conducted in the era of drug-eluting stents (DES), demonstrated that PCI for cor- onary stenosis with FFR $\leq 0.80$ improved patients' clinical outcomes as compared with medical therapy. ${ }^{3,4}$ Subsequently, the FAME 2 substudy for the medical therapy group showed that the rate of vessel-oriented clinical events was significantly greater when lesions had an FFR $\leq 0.80$, regardless of the angiographic appearance of the stenosis. ${ }^{5}$ Therefore, an FFR between 0.75 and 0.80 constitutes a "gray zone" for clinical decision-making, and the indication for revascularization in a case of gray zone FFR is debatable. In the present study, we compared long-term clinical outcomes of PCI using DES vs. medical therapy for coronary stenosis with gray zone FFR.

Received June 11, 2018; revised manuscript received September 1, 2018; accepted September 13, 2018; released online October 13, 2018 Time for primary review: 56 days

Department of Cardiovascular Medicine, Wakayama Medical University, Wakayama (T. Kubo, Y.I., Y.M., Y.S., K.S., T. Kameyama, H.E., Y.K., T.A.); Department of Cardiovascular Medicine, Hashimoto Municipal Hospital, Hashimoto (M.T.); and Department of Cardiovascular Medicine, Shingu Municipal Medical Center, Shingu (K.T., K.M., Y.A., H.K., T.T.), Japan

Mailing address: Takashi Kubo, MD, PhD, Department of Cardiovascular Medicine, Wakayama Medical University, 811-1 Kimiidera, Wakayama 641-8509, Japan. E-mail: takakubo@wakayama-med.ac.jp

ISSN-1346-9843 All rights are reserved to the Japanese Circulation Society. For permissions, please e-mail: cj@j-circ.or.jp 


\section{Methods}

\section{Study Population}

This was a retrospective multicenter study (Retrospective comparison of long-term clinical outcomes between percutaneous coronary intervention and medical therapy in stable coronary artery disease with gray zone fractional flow reserve: COMFORTABLE retrospective study) in Wakayama Medical University Hospital, Wakayama, Japan, and its affiliated hospitals, including Hashimoto Municipal Hospital, Hashimoto, and Shingu Municipal Medical Center, Shingu, Japan. These 3 hospitals are core medical centers in Wakayama Prefecture, Japan. The university physicians rotate through these 3 hospitals and perform coronary catheterization in each hospital. Between June 2005 and December 2016, we identified patients with FFR $0.75-0.80$ in a native coronary artery. The patients with FFR $0.75-0.80$ were categorized into 2 groups according to the therapeutic approach to the target coronary artery: PCI using DES (PCI group) or medical therapy (medical therapy group). During the study period, we used the FFR cutoff value of $<0.75$ for identifying myocardial ischemia. In patients with FFR $0.75-0.80$, therefore, we generally chose medical therapy and carefully selected PCI in reference to the clinical characteristics of the patients (e.g., age, physical activity, and symptoms) and the results of noninvasive tests for ischemia. Exclusion criteria were FFR in a culprit vessel responsible for acute coronary syndrome (ACS), FFR in a vessel treated with PCI in the previous 6 months, and FFR in a vessel treated with coronary artery bypass grafting (CABG). In addition, we excluded patients who underwent PCI for the target coronary lesion but not using DES (i.e., bare-metal stent implantation, balloon angioplasty alone, or CABG). The study was approved by the research ethics board at each participating hospital, and written informed consent was given by all patients.

\section{Data Collection}

Data on patients' clinical characteristics, medications, blood tests, angiography, FFR and PCI were manually extracted after reviewing all the available records. Clinical outcomes were investigated through telephone interview with patients and/or medical records review.

Baseline Clinical Data Baseline clinical data of the patients included age, sex, hypertension (defined as systolic blood pressure (BP) $\geq 140 \mathrm{mmHg}$, diastolic $\mathrm{BP} \geq 90 \mathrm{mmHg}$, or antihypertensive medication), diabetes mellitus (defined as hemoglobin A1c $\geq 6.5 \%$ or antidiabetic medication), dyslipidemia (defined as low-density lipoprotein cholesterol $\geq 140 \mathrm{mg} / \mathrm{dL}$ or antilipidemic medication), current smoker (defined as having smoked at least 100 cigarettes lifetime and smoking currently), and obesity (defined as body mass index $\geq 25 \mathrm{~kg} / \mathrm{m}^{2}$ ), hemodialysis, prior myocardial infarction (MI) (presented as patient-level data), and prior PCI (presented as patient-level data).

Coronary Angiography (CAG) CAG was performed in the standard manner. Reference vessel diameter, minimum lumen diameter (MLD), percent diameter stenosis $[\% \mathrm{DS}=(1-\mathrm{MLD} /$ reference vessel diameter $) \times 100]$, and lesion length were measured using a dedicated software for quantitative $\mathrm{CAG}$ at each participating center. Severe coronary stenosis was defined as a $\% \mathrm{DS}>70 \%$. Tandem lesion was defined as 2 separate stenoses in the same coronary artery, separated by an angiographically normal seg- ment. Long lesion was defined as $\geq 28 \mathrm{~mm}$ in length. Left main coronary artery (LMCA) lesion was defined as any degree of stenosis in the LMCA. Bifurcation lesion was defined as a lesion in a main branch involving side branches $\geq 2 \mathrm{~mm}$ in diameter. Lesion complexity was assessed according to the American college of cardiology/American heart association (ACC/AHA) criteria, and the presence or absence of type $\mathrm{C}$ lesions was noted. ${ }^{6}$

FFR Intracoronary pressure was measured by a pressure-guidewire system (PressureWire, Abbot Vascular, Santa Clara, CA, USA; Smartwire, Philips Volcano, San Diego, CA, USA; Comet, Boston Scientific, Marlborough, MA, USA; or OptoWire, Zeon Medical, Tokyo, Japan). Following pressure zero-calibration and equalization, the pressure-guidewire was placed in the distal part of the target coronary artery. Intracoronary nitroglycerin was administered prior to FFR measurement. FFR was calculated as the mean distal coronary pressure, measured by the pressure-guidewire, divided by the mean aortic pressure, simultaneously measured by the guiding catheter, during maximal hyperemia. Maximal hyperemia was induced by intravenous continuous infusion of adenosine 5'-triphosphate, given at $\geq 150 \mu \mathrm{g} / \mathrm{kg} / \mathrm{min}$ via the cephalic vein of forearm or femoral vein. ${ }^{7}$ The pressure sensor was pulled back in the guiding catheter during maximal hyperemia, and both pressures were checked to exclude any transducer drift.

PCI The decision for PCI or medical therapy was at the operator's discretion. PCI was performed in the standard manner using a DES. The patients who underwent PCI were treated with aspirin and another antiplatelet agent such as clopidogrel or prasugrel for at least 6 months after PCI.

Clinical Outcomes The primary endpoint was target vessel failure (TVF), defined as a composite of cardiac death, MI and ischemia-driven target vessel revascularization (TVR). Secondary endpoints included the individual components of the primary endpoint, a composite of cardiac death or MI; clinically-driven TVR; urgent TVR; and stroke. Cardiac death was defined as any death not clearly attributable to a non-cardiac cause. ${ }^{8}$ MI was defined as a clinical episode of typical chest pain with development of new $Q$ waves in $\geq 2$ contiguous leads on ECG or elevation of the creatine kinase myocardial band (CK-MB) fraction (spontaneous: > upper reference limit, periprocedural: $>3$-fold upper reference limit), either of which was attributed to the target coronary artery. ${ }^{8}$ TVR was defined as subsequent revascularization of the target vessel by either PCI (additional stent or angioplasty) or CABG. TVR was considered ischemia-driven if revascularization was performed on a patient who had ischemic signs confirmed by noninvasive tests or FFR reassessment (with an inducible ischemia cutoff set at $<0.75$ ), despite the presence or absence of ischemic symptoms. ${ }^{9}$ TVR was considered to be clinically-driven if revascularization was performed on a patient who had ischemic signs confirmed by noninvasive tests or FFR reassessment and clinical symptoms such as chest pain that increased in frequency, intensity or duration. ${ }^{9}$ TVR was considered to be urgent if a patient was admitted to the hospital with persistent or increasing chest pain (with or without ST-segment or T-wave changes or elevated cardiac biomarkers) and the revascularization procedure was performed during the same hospitalization. ${ }^{4}$ In cases of patients without ST-segment or T-wave changes and elevated cardiac biomarker levels, urgent TVR was performed according to FFR $<0.75$. Severity of chest pain 


\begin{tabular}{|c|c|c|c|}
\hline & $\begin{array}{c}\mathrm{PCl} \\
(\mathrm{n}=78)\end{array}$ & $\begin{array}{l}\text { Medical therapy } \\
\quad(n=185)\end{array}$ & $P$ value \\
\hline Age, years & $71 \pm 9$ & $69 \pm 9$ & 0.150 \\
\hline Male & $63(81)$ & $137(74)$ & 0.244 \\
\hline Hypertension & $64(82)$ & $147(79)$ & 0.563 \\
\hline Diabetes mellitus & $35(45)$ & $86(46)$ & 0.807 \\
\hline Dyslipidemia & $59(76)$ & $148(80)$ & 0.431 \\
\hline Current smoker & $21(27)$ & $57(31)$ & 0.528 \\
\hline Obesity & $16(21)$ & $47(25)$ & 0.448 \\
\hline Hemodialysis & $1(1)$ & $10(5)$ & 0.183 \\
\hline Prior MI & $21(27)$ & $72(39)$ & 0.063 \\
\hline Prior $\mathrm{PCl}$ & $40(51)$ & $117(63)$ & 0.071 \\
\hline Vessel & & & 0.250 \\
\hline LAD & $54(69)$ & $124(67)$ & \\
\hline LCX & $15(19)$ & $26(14)$ & \\
\hline $\mathrm{RCA}$ & $9(12)$ & $35(19)$ & \\
\hline \multicolumn{4}{|l|}{ Stenosis } \\
\hline Reference vessel diameter, $\mathrm{mm}$ & $2.70 \pm 0.56$ & $2.61 \pm 0.58$ & 0.247 \\
\hline Minimum lumen diameter, mm & $1.29 \pm 0.49$ & $1.30 \pm 0.39$ & 0.861 \\
\hline Diameter stenosis, \% & $52 \pm 15$ & $50 \pm 13$ & 0.227 \\
\hline Severe stenosis (\%DS > $70 \%$ ) & $15(19)$ & $9(5)$ & 0.004 \\
\hline Lesion length, mm & $17 \pm 8$ & $17 \pm 7$ & 0.999 \\
\hline \multicolumn{4}{|l|}{ Lesion characteristics } \\
\hline Tandem & $12(15)$ & $24(13)$ & 0.752 \\
\hline Long (length $\geq 28 \mathrm{~mm}$ ) & $12(15)$ & $20(11)$ & 0.301 \\
\hline LMCA & $1(1)$ & $11(6)$ & 0.117 \\
\hline Bifurcation & $18(23)$ & $39(21)$ & 0.718 \\
\hline ACC/AHA type $\mathrm{C}$ lesions & $15(19)$ & $25(14)$ & 0.238 \\
\hline FFR & $0.78(0.76-0.79)$ & $0.78(0.77-0.79)$ & 0.070 \\
\hline
\end{tabular}

Values are given as $n(\%)$, mean \pm standard deviation, or median (interquartile range). ACC/AHA=American College of Cardiology/American Heart Association; ACS, acute coronary syndrome; FFR, fractional flow reserve; LAD, left anterior descending coronary artery; LCX, left circumflex coronary artery; LMCA, left main coronary artery; MI, myocardial infarction; $\mathrm{PCl}$, percutaneous coronary intervention; RCA, right coronary artery.

was determined according to the Canadian Cardiovascular Society (CCS) Angina Grading Scale. ${ }^{10}$ Stroke was defined as a sudden onset of neurological deficit resulting from vascular lesions of the brain and persisting for $>24 \mathrm{~h}$.

\section{Statistical Analysis}

Statistical analysis was performed using JMP13.0 (SAS Institute, Cary, NC, USA). Categorical variables are presented as frequency and percentage and were compared by chi-squared test or Fisher's exact test (if an expected cell count was $<5$ ). Continuous variables are presented as mean \pm standard deviation and were compared by unpaired Student's t-test. The FFR values and follow-up periods are presented as medians and interquartile ranges (IQRs) and compared using the Mann-Whitney $\mathrm{U}$ test (between-group comparison). Kaplan-Meier curves were constructed for clinical outcomes. The Cox proportional hazards model was used to calculate hazard ratio (HR) and 95\% confidence intervals $(95 \% \mathrm{CI})$ for the between-group comparisons of clinical outcome. The consistency of treatment effects across subgroups defined according to the baseline clinical and angiographic characteristics was assessed by Cox proportional hazards model with the test for interaction. $\mathrm{P}<0.05$ was considered statistically significant.

\section{Results}

\section{Patients' Characteristics}

During the study period, 2,011 patients underwent FFR. From these, we identified 267 patients who had a coronary artery presenting with FFR $0.75-0.80$. We excluded 4 patients from the analysis because of PCI using a baremetal stent $(n=1)$ or drug-coated balloon $(n=3)$. Thus, 263 patients constituted the final study population: 78 patients in the PCI group and 185 patients in the medical therapy group. There were no patients who had $\geq 2$ vessels with FFR $0.75-0.80$. The median FFR value in the PCI group and the medical therapy group was 0.78 (IQR $0.76-0.79$ ) and 0.78 (IQR 0.77-0.79), respectively $(\mathrm{P}=0.070)$. Baseline clinical and angiographic characteristics were not different between the PCI and medical therapy groups, except for severe stenosis lesion ( $19 \%$ vs. $5 \%, \mathrm{P}=0.004)$ (Table 1). The DES in the PCI group included 31 Xience (Abbot Vascular), 17 Cypher (Cordis, Miami, FL, USA), 11 Ultimaster (Terumo, Tokyo, Japan), 9 Synergy (Boston Scientific), 5 Promus (Boston Scientific), 3 Nobori (Terumo), 1 Taxus (Boston Scientific), and 1 Resolute Integrity (Medtronic, Santa Rosa, CA, USA). 


\begin{tabular}{lccc|}
\hline \multicolumn{1}{l}{ Table 2. Medications and Blood Test Results at Follow-up } & & \\
& $\begin{array}{c}\mathbf{P C l} \\
\mathbf{( n = 7 8 )}\end{array}$ & $\begin{array}{c}\text { Medical therapy } \\
(\mathbf{n}=\mathbf{1 8 5})\end{array}$ & P value \\
Medications & $60(77)$ & $146(79)$ & 0.720 \\
Aspirin & $23(29)$ & $26(14)$ & 0.003 \\
Thienopyridine & $25(32)$ & $80(43)$ & 0.091 \\
$\beta$-blocker & $49(63)$ & $103(56)$ & 0.284 \\
ACEl/ARB & $45(58)$ & $109(59)$ & 0.854 \\
Statin & $25(32)$ & $55(30)$ & 0.709 \\
Oral antiglycemic agents & $7(9)$ & $22(12)$ & 0.490 \\
Insulin & $168 \pm 36$ & & \\
Blood test results & $46 \pm 12$ & $163 \pm 34$ & 0.286 \\
Total cholesterol, mg/dL & $93 \pm 30$ & $45 \pm 13$ & 0.561 \\
HDL cholesterol, mg/dL & $148 \pm 108$ & $89 \pm 26$ & 0.278 \\
LDL cholesterol, mg/dL & $6.2 \pm 0.9$ & $130 \pm 81$ & 0.139 \\
Triglyceride, mg/dL & $6.2 \pm 1.1$ & 0.999 \\
HbA1c, \% & & & \\
\hline
\end{tabular}

Values are given as $\mathrm{n}(\%)$. ACEI, angiotensin-converting-enzyme inhibitor; ARB, angiotensin II receptor blocker; FFR, fractional flow reserve; HbA1c, hemoglobin A1c; HDL, high density lipoprotein cholesterol; LDL, low-density lipoprotein cholesterol; $\mathrm{PCl}$, percutaneous coronary intervention.

\begin{tabular}{|lcccc|}
\hline Table 3. Clinical Outcomes & PCl & $\begin{array}{c}\text { Medical therapy } \\
(\mathbf{n = 1 8 5}\end{array}$ & HR (95\% Cl) & P value \\
TVF & $\mathbf{( n = 7 8 )}$ & $35(19)$ & $0.33(0.13-0.84)$ & 0.008 \\
Cardiac death or MI & $5(6)$ & $4(2)$ & $0.61(0.07-5.49)$ & 0.645 \\
Cardiac death & $1(1)$ & $2(1)$ & - & - \\
MI & $0(0)$ & $2(1)$ & $1.16(0.11-12.87)$ & 0.903 \\
Ischemia-driven TVR & $1(1)$ & $33(18)$ & $0.28(0.10-0.79)$ & 0.005 \\
Clinically-driven TVR & $4(5)$ & $23(12)$ & $0.20(0.05-0.87)$ & 0.008 \\
Urgent TVR & $2(3)$ & $18(10)$ & $0.27(0.07-0.98)$ & 0.039 \\
Stroke & $2(3)$ & $4(2)$ & $0.70(0.08-6.32)$ & 0.744 \\
\hline
\end{tabular}

Values are given as $\mathrm{n}(\%)$. $\mathrm{Cl}$, confidence interval; $\mathrm{HR}$, hazard ratio; $\mathrm{Ml}$, myocardial infarction; $\mathrm{PCl}$, percutaneous coronary intervention; TVF, target vessel failure; TVR, target vessel revascularization.

PCI

A stent was successfully implanted in all patients of the PCI group; 1 patient had angiographic slow-flow during the PCI but no significant CK-MB elevation was found immediately after the procedure. There were no side branch occlusions or coronary dissections requiring additional procedures.

\section{Clinical Outcomes}

The median follow-up period in the PCI and medical therapy groups was 3.0 (IQR 2.1-8.1) years and 3.9 (IQR 2.5-7.3) years, respectively $(\mathrm{P}=0.110)$. Medications at follow-up did not differ between groups, except for thienopyridines ( $29 \%$ vs. $14 \%, \mathrm{P}=0.003$ ) (Table 2). Blood test results at follow-up were comparable between groups (Table 2).

The frequency of TVF was significantly lower in the PCI group compared with the medical therapy group ( $6 \%$ vs. 19\%, HR: $0.33,95 \%$ CI: $0.13-0.84, \mathrm{P}=0.008$ ) (Table 3, Figure 1). The frequency of a composite of cardiac death or MI was not different between groups ( $1 \%$ vs. $2 \%$, HR: $0.61,95 \%$ CI: $0.07-5.49, \mathrm{P}=0.645$ ) (Table 3, Figure 2). The frequency of ischemia-driven TVR ( $5 \%$ vs. $18 \%$, HR: 0.28 ,

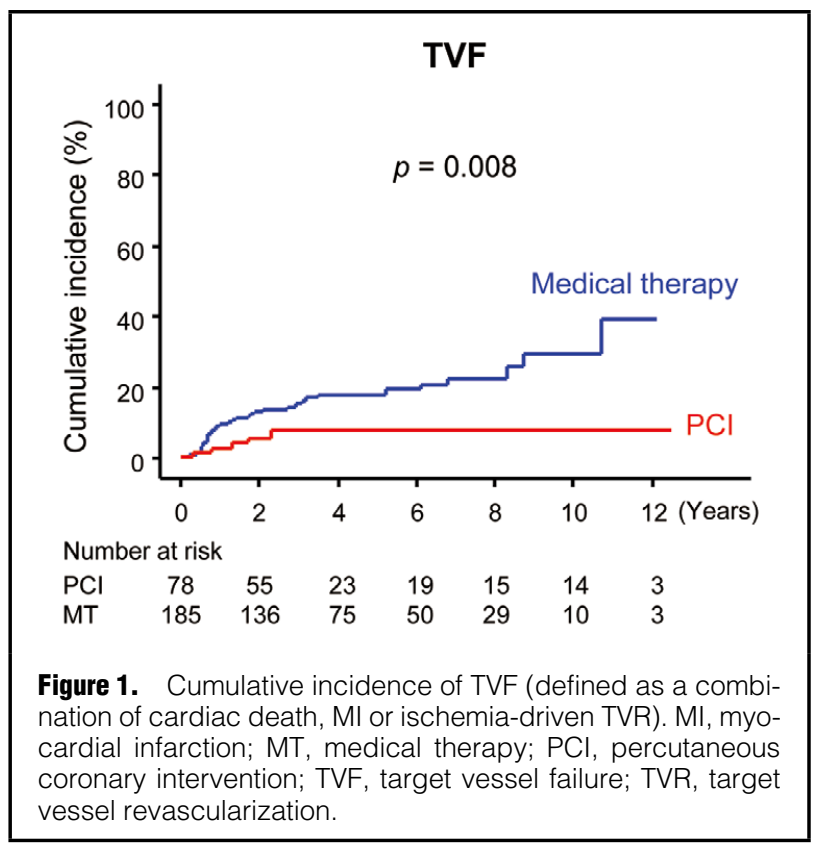



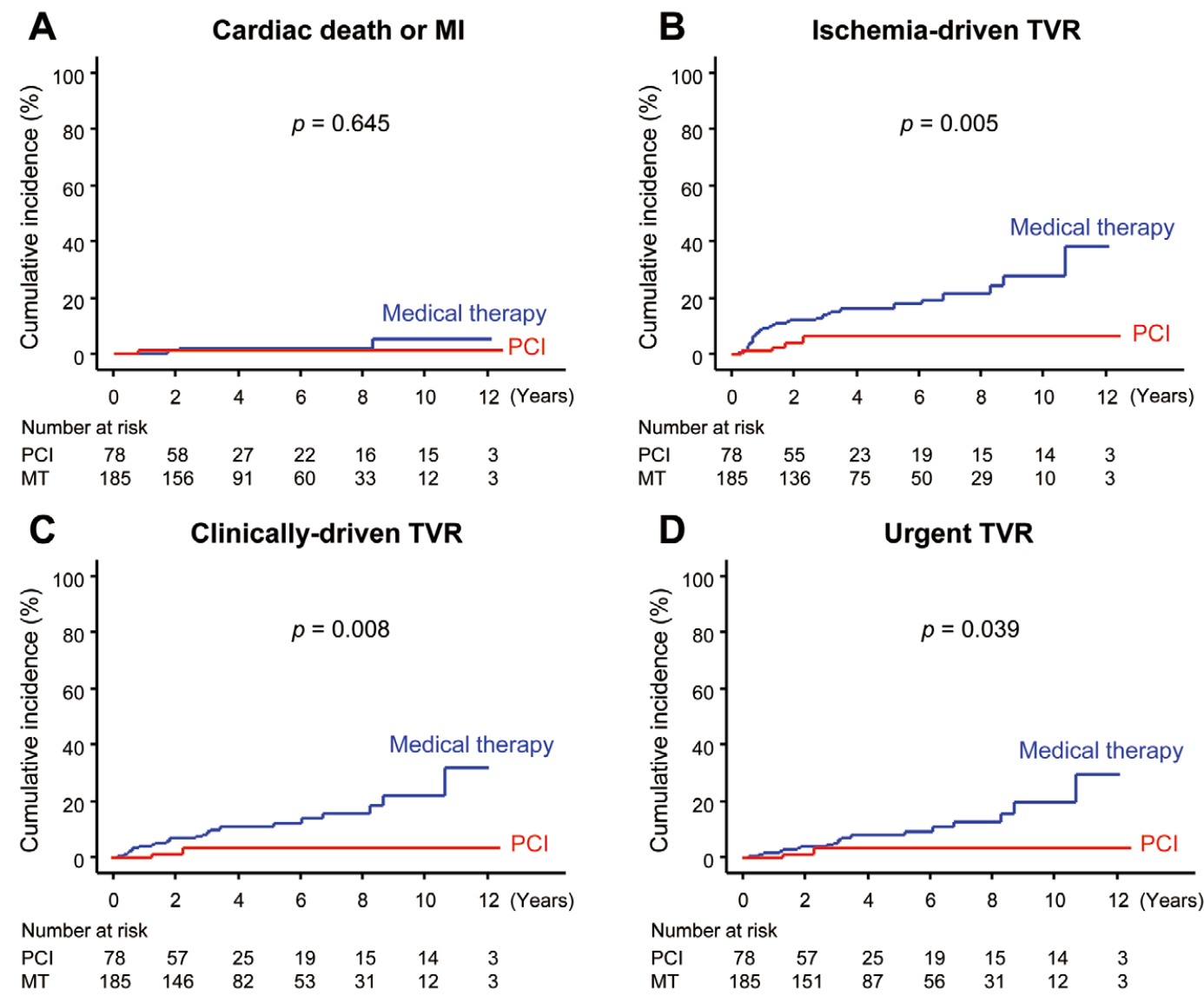

Figure 2. Cumulative incidence of cardiac death or MI (A), ischemia-driven TVR (B), clinically-driven TVR (C), and urgent TVR (D). Abbreviations as in Figure 1.

95\% CI: $0.10-0.79, \mathrm{P}=0.005)$, clinically-driven TVR $(3 \%$ vs. $12 \%$, HR: $0.20,95 \% \mathrm{CI}: 0.05-0.87, \mathrm{P}=0.008)$, and urgent TVR (3\% vs. $10 \%$, HR: $0.27,95 \%$ CI: $0.07-0.98$, $\mathrm{P}=0.039)$ was significantly lower in the PCI group compared with the medical therapy group (Table 3, Figure 2). The frequency of stroke was not different between the 2 groups ( $1 \%$ vs. $2 \%$, HR: $0.70,95 \%$ CI: $0.08-6.32, \mathrm{P}=0.744)$ (Table 3).

\section{Clinical Presentations at TVR}

The frequency of ischemia-driven TVR related to a decrease in FFR $(<0.75)$ was 0 of $4(0 \%)$ in the PCI group and 16 of $33(48 \%)$ in the medical therapy group (FFR, $0.69 \pm 0.05)$ $(\mathrm{P}=0.118)$. There was no deferral of TVR in patients who had FFR $<0.75$ or positive results for noninvasive ischemia tests with or without ischemic symptoms. In patients with clinically-driven TVR, the distribution of CCS class I/II/III/IV at TVR was $0 / 0 / 0 / 2$ in the PCI group and 3/6/6/8 in the medical therapy group $(\mathrm{P}=0.353)$; though there was no ischemic symptoms at baseline in either group. The frequency of urgent TVR related to chest pain with ST-segment or T-wave changes on admission ECG was 2 of $2(100 \%)$ in the PCI group and 13 of $18(72 \%)$ in the medical therapy group $(\mathrm{P}=0.999)$. The frequency of urgent TVR related to chest pain with elevated cardiac biomark- ers was 1 of $2(50 \%)$ in the PCI group and 5 of $18(28 \%)$ in the medical therapy group $(\mathrm{P}=0.521)$.

\section{TVR at Scheduled Angiographic Follow-up}

In the PCI group, scheduled angiographic follow-up at 6-12 months after FFR measurement was performed in 61 $(78 \%)$ patients. The FFR reassessment and/or noninvasive ischemia tests was performed in 19 patients and 42 patients, respectively. There was 1 case of ischemia-driven TVR but none of clinically-driven TVR.

In the medical therapy group, the scheduled angiographic follow-up was performed in $40(22 \%)$ patients (vs. the PCI group, $\mathrm{P}<0.001)$. The FFR reassessment and/or noninvasive ischemic tests was performed in 25 patients and 15 patients, respectively. There were 10 cases of ischemia-driven TVR and 6 of clinically-driven TVR.

\section{Subgroup Analysis}

As for the reduction in TVF, the tests for interactions showed that the treatment effect of PCI compared with medical therapy was consistent across the subgroups with respect to age $(\mathrm{P}=0.762)$, current smoker $(\mathrm{P}=0.679)$, prior $\mathrm{MI}(\mathrm{P}=0.151)$, prior $\mathrm{PCI}(\mathrm{P}=0.378), \mathrm{LAD}$ lesion $(\mathrm{P}=0.421)$, and severe stenosis lesion $(\mathrm{P}=0.258)$, but there were significant heterogeneities across the subgroups with respect to 


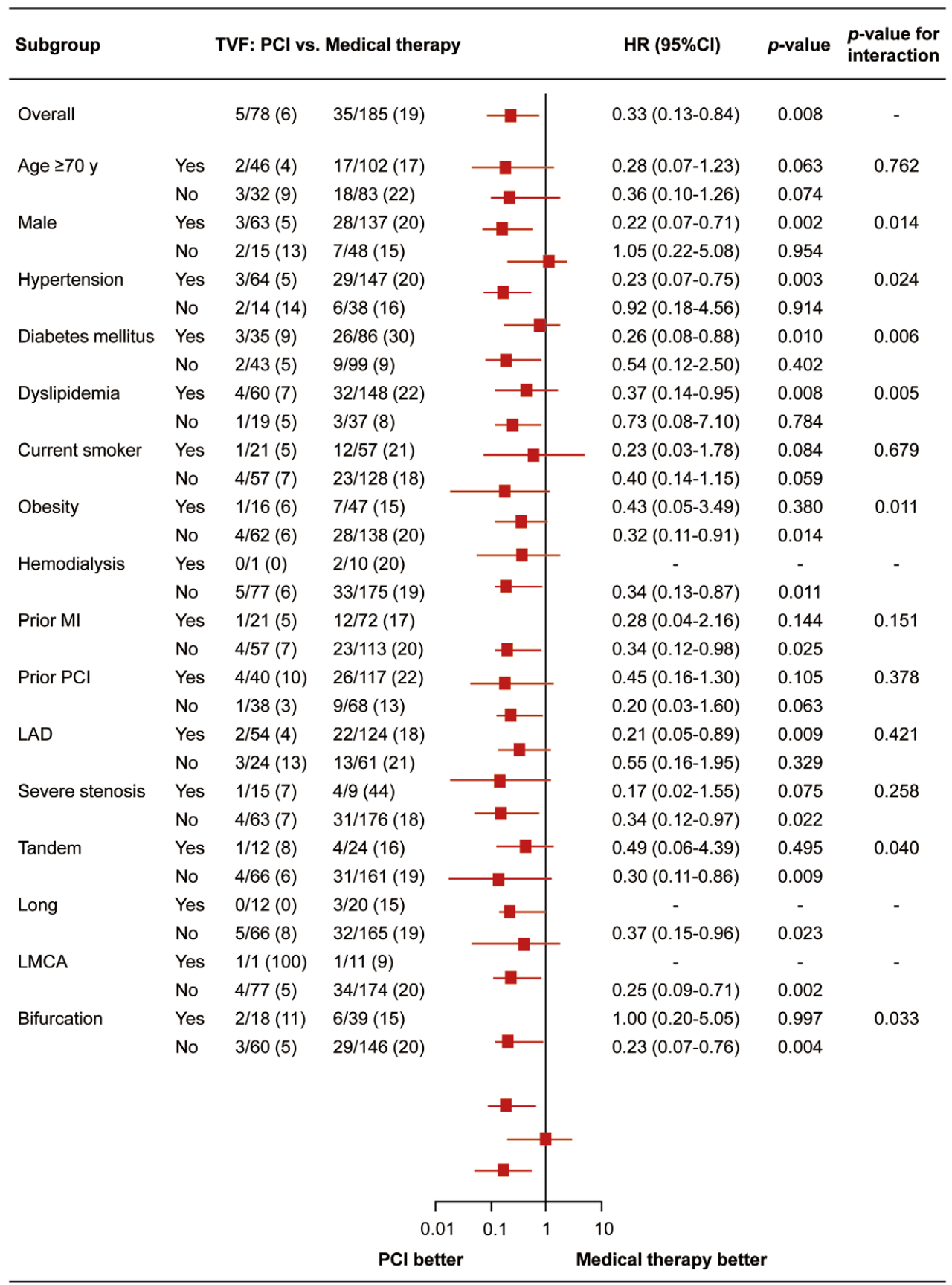

Figure 3. Subgroup analysis. Subgroup analyses with $\mathrm{HR}$ and $95 \% \mathrm{Cl}$ are shown for TVF among subgroups of patients who underwent $\mathrm{PCl}$ or medical therapy. The frequency of TVF is shown as n/total $\mathrm{n}$. (\%). The $\mathrm{P}$ value for interaction represents the likelihood of interaction between the variable and the relative treatment effect. $\mathrm{Cl}$, confidence interval; HR, hazard ratio; LAD, left anterior descending coronary artery; LMCA, left main coronary artery; MI, myocardial infarction; PCI, percutaneous coronary intervention; TVF, target vessel failure.

sex $(\mathrm{P}=0.014)$, hypertension $(\mathrm{P}=0.024)$, diabetes mellitus $(\mathrm{P}=0.006)$, dyslipidemia $(\mathrm{P}=0.005)$, obesity $(\mathrm{P}=0.011)$, tandem lesion $(\mathrm{P}=0.040)$, and bifurcation lesion $(\mathrm{P}=0.033)$ (Figure 3).

\section{Discussion}

The main finding of the present study comparing long- term clinical outcomes in patients with gray zone FFR between PCI using DES and medical therapy was the significantly lower frequency of TVF in the PCI group. The reason for the lower frequency of TVF in the PCI group was mainly the reduction in the frequency of angina or myocardial ischemia. The frequency of cardiac death or MI was not different between the 2 groups. 


\section{FFR-Guided PCI}

Both the DEFER and the FAME study showed that FFRguided decision-making for revascularization was associated with a favorable clinical outcome., ${ }^{2,3}$ Subsequently, the FAME 2 study demonstrated that in functionally significant stenoses, PCI compared with medical therapy decreased the need for urgent revascularization, though the frequency of death or MI was not different between PCI and medical therapy. ${ }^{4}$ In the FAME 2 study, however, patient enrollment was interrupted early after interim analyses by the data and safety monitoring board, and so the small number of patients may have biased the results. Therefore, the clinical effect of PCI with FFR $\leq 0.80$ is still being debated.

\section{Clinical Outcomes in FFR $\mathbf{0 . 7 5 - 0 . 8 0}$ vs. FFR $>0.80$}

A recent meta-analysis of FFR studies demonstrated that major adverse clinical events increased as FFR decreased in patients treated medically (median follow-up $\approx 16$ months). ${ }^{11}$ Previously, we also showed that medically treated patients with FFR $0.75-0.80(n=56)$ were at higher risk of TVF for 3 years than those with FFR $>0.80$ $(n=94) .{ }^{12}$ Hence, for patients with gray zone FFR, further therapeutic intervention in addition to medical therapy might be warranted to improve clinical outcomes.

\section{PCI vs. Medical Therapy}

A few non-randomized studies have demonstrated the safety of deferral of PCI (i.e., medical therapy) in patients with gray zone FFR, though the studies were relatively small, with low-risk patients who had predominantly single-vessel disease. ${ }^{11}$ Also, a subanalysis of the IRIS-FFR (Interventional Cardiology Research In-cooperation Society Fractional Flow Reserve) registry disclosed no significant difference in major adverse clinical events between PCI and medical therapy in patients with gray zone FFR. ${ }^{9}$ In contrast, some retrospective studies, including the present study, have shown that PCI compared with medical therapy was associated with better clinical outcomes in patients with gray zone FFR. ${ }^{13,14}$ To prove the superiority of PCI compared with medical therapy in patients with gray zone FFR, we are planning a prospective, multicenter randomized study (the COMFORTABLE prospective study, UMIN000031526). The results of this prospective study will settle the controversy of gray zone FFR.

\section{Reduction in Death or MI by PCI}

A large-scale single-center study showed that the patients with single-vessel disease and a coronary stenosis with gray zone FFR had a half as much risk of the composite of death and MI for up to 5 years if treated with PCI instead of medical therapy. ${ }^{13} \mathrm{~A}$ recent retrospective study also demonstrated that the composite of death or MI was significantly lower in PCI. ${ }^{14}$ However, in most studies, including the present study, the reduction in major adverse clinical events in PCI compared with medical therapy was mainly driven by the reduction in the frequency of angina or myocardial ischemia without any difference in the frequency of death or MI.9,11 In terms of the reduction in the composite of death or MI, PCI may offer a benefit in patients with more severe FFR values than gray zone FFR.,11

\section{Reduction in Urgent TVF by PCI}

In the present study, the frequency of urgent TVR was significantly lower in the PCI group compared with the medical therapy group. Urgent TVR is considered to be an important surrogate marker of ACS/MI. However, urgent TVR is an endpoint based on subjective evaluation of acute ischemic symptoms and does not necessarily require objective evidence of coronary thrombosis. Therefore, patients who underwent urgent TVR might include not only ACS/MI caused by coronary thrombosis but also new or worsening angina pectoris caused by plaque progression and lumen narrowing. Further study is needed to assess whether PCI in lesions presenting gray zone FFR contribute to reducing future ACS/MI.

\section{Subgroup Analysis}

In the present study, there were differences in the treatment effect across subgroups defined according to the baseline clinical and angiographic characteristics. The benefit of PCI was greater in patients with traditional coronary risk factors including male sex, hypertension, diabetes mellitus, dyslipidemia, and obesity, whereas it was smaller in patients with complex lesions including tandem lesions and bifurcation lesions. However, as the sample size was small for most subgroups, caution is warranted in the interpretation of our results and in drawing conclusions from them.

\section{Study Limitations}

First, this was a retrospective study. The decision on whether to measure FFR was made by the operators. Also, medical therapy was more often selected than PCI. Hence, there might be a selection bias. Second, the fact that we were able to collect limited amounts of information for individual patients (via chart review) and also that the number of studied patients was at most, moderate in size, precludes performing propensity score matching to control for confounding. Third, FFR pullback data were not available in the present study. Only absolute FFR was used for analysis. Fourth, the medical therapy during follow-up period may have been suboptimal. However, our results are reflective of real-world practice. Fifth, $40(22 \%)$ patients in the medical therapy group underwent the scheduled angiographic follow-up. Of these, 10 patients had ischemia-driven TVR. The scheduled angiographic follow-up may have increased the incidence of deferred lesion revascularization. Finally, the present study was underpowered to assess a group difference in death or MI because of the low frequency of each event.

\section{Conclusions}

In patients with gray zone FFR, compared with medical therapy, PCI decreased the frequency of TVF, which was mainly driven by a reduction in the frequency of angina or myocardial ischemia without any difference in the frequency of cardiac death or MI.

\section{Acknowledgment}

We thank Mariko Uemura for assistance with clinical data collection.

\section{Disclosures}

Funding Sources: None.

Conflicts of Interest: T. Kubo has received lecture fees from Abbot Vascular. Y.S. received lecture fees from Abbot Vascular and Philips Volcano. T.A. has received lecture fees from Abbot Vascular, Philips Volcano, and Boston Scientific, and research grants from Abbot Vascular, Philips Volcano, Boston Scientific, and Zeon Medical. All other authors have no relationships relevant to the contents of this paper to disclose. 


\section{References}

1. Pijls NH, De Bruyne B, Peels K, van der Voort PH, Bonnier HJ, Bartunek J, et al. Measurement of fractional flow reserve to assess the functional severity of coronary-artery stenoses. $N \mathrm{Engl}$ J Med 1996; 334: 1703-1708.

2. Zimmermann FM, Ferrara A, Johnson NP, van Nunen LX, Escaned J, Albertsson P, et al. Deferral vs. performance of percutaneous coronary intervention of functionally non-significant coronary stenosis: 15-year follow-up of the DEFER trial. Eur Heart $J$ 2015; 36: 3182-3188.

3. van Nunen LX, Zimmermann FM, Tonino PA, Barbato E, Baumbach A, Engstrøm T, et al. Fractional flow reserve versus angiography for guidance of PCI in patients with multivessel coronary artery disease (FAME): 5-year follow-up of a randomised controlled trial. Lancet 2015; 386: 1853-1860.

4. De Bruyne B, Pijls NH, Kalesan B, Barbato E, Tonino PA, Piroth Z, et al. Fractional flow reserve-guided PCI versus medical therapy in stable coronary disease. N Engl J Med 2012; 367: $991-1001$.

5. Ciccarelli G, Barbato E, Toth GG, Gahl B, Xaplanteris P, Fournier S, et al. Angiography versus hemodynamics to predict the natural history of coronary stenoses: Fractional flow reserve versus angiography in multivessel evaluation 2 substudy. Circulation 2018; 137: 1475-1485.

6. Ellis SG, Vandormael MG, Cowley MJ, DiSciascio G, Deligonul U, Topol EJ, et al. Coronary morphologic and clinical determinants of procedural outcome with angioplasty for multivessel coronary disease: Implications for patient selection. Multivessel Angioplasty Prognosis Study Group. Circulation 1990; 82: 1193 1202 .
7. Emori H, Kubo T, Kameyama T, Ino Y, Matsuo Y, Kitabata H, et al. Diagnostic accuracy of quantitative flow ratio for assessing myocardial ischemia in prior myocardial infarction. Circ J 2018; 82: $807-814$.

8. Cutlip DE, Windecker S, Mehran R, Boam A, Cohen DJ, van Es GA, et al. Academic Research Consortium, clinical end points in coronary stent trials: A case for standardized definitions. Circulation 2007; 115: $2344-2351$

9. Kang DY, Ahn JM, Lee CH, Lee PH, Park DW, Kang SJ, et al. Deferred vs. performed revascularization for coronary stenosis with grey-zone fractional flow reserve values: Data from the IRIS-FFR registry. Eur Heart J 2018; 39: 1610-1619.

10. Campeau L. Grading of angina pectoris (Letter). Circulation 1976; 54: 522-523

11. Johnson NP, Tóth GG, Lai D, Zhu H, Açar G, Agostoni P, et al. Prognostic value of fractional flow reserve: Linking physiologic severity to clinical outcomes. J Am Coll Cardiol 2014; 64: $1641-1654$.

12. Shiono Y, Kubo T, Tanaka A, Ino Y, Yamaguchi T, Tanimoto $\mathrm{T}$, et al. Long-term outcome after deferral of revascularization in patients with intermediate coronary stenosis and gray-zone fractional flow reserve. Circ J 2015; 79: 91-95.

13. Adjedj J, De Bruyne B, Floré V, Di Gioia G, Ferrara A, Pellicano $\mathrm{M}$, et al. Significance of intermediate values of fractional flow reserve in patients with coronary artery disease. Circulation 2016; 133: $502-508$.

14. Agarwal SK, Kasula S, Edupuganti MM, Raina S, Shailesh F, Almomani A, et al. Clinical decision-making for the hemodynamic "gray zone" (FFR 0.75-0.80) and long-term outcomes. $J$ Invasive Cardiol 2017; 29: 371-376. 